\title{
COMPARATIVE STUDY OF THE SHEAR BOND STRENGTH OF ZIRCONIA-BASED DENTAL CERAMICS TO RESIN CEMENTS AFTER CHEMO-MECHANICAL SURFACE TREATMENT
}

\author{
Paulina Łagodzińska' ${ }^{1}$ Kinga Bociong ${ }^{2}$, Jerzy Sokołowski ${ }^{2,3}$, Beata Dejak ${ }^{1}$ \\ 'Department of Prosthodontics, Medical University of Lodz, Poland \\ ${ }^{2}$ University Laboratory of Material Research, Medical University of Lodz, Poland \\ ${ }^{3}$ Department of General Dentistry, Medical University of Lodz, Poland
}

\begin{abstract}
INTRODUCTION: The loss of retention of zirconia-based fixed partial dentures (FPDs) is one of the most frequently reported failures.

ОвJECTIVEs: The purpose of this study was to evaluate the shear bond strength (SBS) of four resin cements to zirconia after air-borne particle abrasion with alumina of different grades and application of respective primers incorporating organophosphate monomers.

MATERIAL AND METHODS: The 443 cuboid-shape zirconia specimens (Ceramill Zi; Amann Girrbach AG) were divided into 16 research groups, according to the chemo-mechanical modification: air-abrasion with alumina particles of $50 \mu \mathrm{m}, 110 \mu \mathrm{m}$ or $250 \mu \mathrm{m}$ grade, no treatment and application of primers and resin cements: Clearfil Ceramic Primer/Panavia F2.0 (Kuraray Medical), Monobond Plus/Multilink Automix (Ivoclar Vivadent AG), AZ-Primer/ResiCem (Shofu), Z-Prime Plus/Duo-Link (Bisco). After storage in $37 \pm 1^{\circ} \mathrm{C}$ distilled water for 24 hours, the SBS test was conducted (Zwick/Roell Z005; Zwick GmbH\&Co.KG) at $1 \mathrm{~mm} / \mathrm{min}$ crosshead speed to failure. Specimens were subjected to fractographic analysis at $\times 2.5$ and $\times 35$ magnification (Seliga Auctus; Seliga Microscopes Sp.z o.o.; SEM S-4700; Hitachi High-Technologies Corporation). Statistical significance was established with the Wilcoxon signed-rank test and the Kruskal-Wallis one-way analysis of variance $(p=0.05)$.

RESULTs: The highest SBS values of zirconia to resin cement were obtained for Z-Prime Plus/DuoLink (8.24-14.15 MPa) and after $50 \mu \mathrm{m}$ alumina air-abrasion $(6.87-14.15 \mathrm{MPa})$. However, the alumina particle grade was insignificant. A significant decrease in the SBS was observed for untreated specimens (4.60-8.24 MPa). The failure mode in untreated specimens was mainly adhesive, while in sandblasted it was cohesive and mixed.

ConcLusions: The chemo-mechanical treatment of zirconia surface before adhesive cementation is crucial to obtain satisfactory values of shear bond strength to resin cements.
\end{abstract}

KEY WORDS: zirconia, resin cement, shear bond strength, aluminum oxide, air-borne particle abrasion.

J Stoma 2019; 72, 4: 158-166

DOI: https://doi.org/10.5114/jos.2019.91233

\section{JOURNAL OF STOMATOLOGY CZASOPISMO STOMATOLOGICZNE

AdDRESS FOR CORRESPONDENCE: Paulina Łagodzińska, Department of Prosthodontics, Medical University of Lodz, 251 Pomorska St., 92-213 Łódź, Poland, e-mail: lagodzinskapaulina@gmail.com

ReCeIved: 25.09.2019 • ACCEPTED: 10.10.2019 • Published: 15.11.2019 


\section{INTRODUCTION}

Zirconia-based dental ceramics are widely used in prosthodontics due to their aesthetics, biocompatibility and outstanding mechanical properties. Five-year clinical observations indicate that the survival rate of zirconia-based fixed partial dentures (FPDs) reaches $73.9-100 \%[14,17]$. The most common failures reported were chipping of the veneering ceramic, loss of retention, framework fractures and secondary caries [14, 17, 29]. Framework fractures and secondary caries resulted mainly from improper handling of the material, such as uneven or too thin framework thickness or improper gingival margin adaptation, whereas the issue of ceramic veneer chipping or loss of retention may be caused by zirconia properties that lead to bonding failures. 3Y-TZP (3 mol\% yttria stabilized tetragonal zirconia polycrystals) is a chemically inert material with smooth surface and dense microstructure composed of crystals without silica or any substantial glassy phase [4, 21, 29].

Bonding failure may occur because of inappropriate choice of the luting agent or inappropriate zirconia surface modification before the cementation procedure. Traditional acid-base cements such as zinc-phosphate, zinc-polycarboxylate, glass-ionomer and resin-modified glass-ionomer cements can be used, but resin cements are preferable $[6,11,23]$. They ensure higher bond strengths, low water solubility, better mechanical properties, lower viscosity, better aesthetics, and lower risk of micro-leakage $[6,11,21]$. Self-etch and dual-cure resin cements are recommended for use in the case of zirconia-based ceramic FPDs. However, the adhesive cementation process is complex and requires the proper zirconia surface modification before the application of the bonding agents. The bond strength to resin cements obtained for the other types of ceramics is better than for zirconia-based ceramics $[12,21]$. Bonding to zirconia restorations is difficult due to its chemical inertness and microstructure - the crystalline phase without the glassy phase $[4,6$, 21,29 ]. The proper zirconia surface preparation is then the significant factor improving the strength and durability of the bonding. There are mechanical and chemical types of modifications recommended, i.e. grinding, airborne particle abrasion with alumina particles, silica coating, selective infiltration etching technique, experimental hot etching solution, laser preparation, MVD (molecular vapor deposition), melted glass micropearl deposition, conventional or experimental primers application, etc. $[1,2,5,8,13,20,21,24]$. There are no long-term observations of the influence of these modifications on the properties of zirconia. It is not known, either, which method is the most effective and harmless. The method of choice effective and easy to perform in every dental office - is air abrasion with alumina particles combined with the use of conventional primers including the 10-MDP monomer (10-methacryloyloxydecyl dihydrogen phosphate) $[2,7$, $13,18,22,25,27,28]$.
It is proven that conventional primers containing $10-\mathrm{MDP}$ monomer are more effective in bonding to zirconia-based ceramics than other primers $[2,7,8,12,13$, $18,24,26,27]$. Phosphate monomers exhibit chemical affinity for zirconium oxide $[8,13,24]$. However, such primer bonding efficacy is satisfactory when combined with air-borne particle abrasion with alumina particles $[2,5,7,13,16,18,22,27,28]$. Omitting air abrasion results in a decrease in bond strength, even bonding failure $[2,5,16,18,22,25,27]$.

There is still no clear algorithm stating the cementation procedure of zirconia-based ceramic FPDs. It is not clearly defined which grade of alumina particle used for air-abrasion is the most effective in improving the bond strength of resin cements to 3Y-TZP, but also the least destructive to its surface, and also which primer along with resin cement ensures the highest bond strength values. The aim of this study was to evaluate the efficacy of air-borne abrasion with alumina particle of different grades and the application of four primers with respect to the shear bond strength of zirconia-based ceramic to resin cements.

\section{OBJECTIVES}

The purpose of this study was to evaluate the shear bond strength (SBS) of four resin cements to zirconia after air-borne particle abrasion with alumina particles of different grade and application of respective primers incorporating organophosphate monomers.

\section{MATERIAL AND METHODS}

\section{SPECIMEN PREPARATION}

The blanks of commercial 3Y-TZP ceramics were used in this study (Ceramill Zi; Amann Girrbach AG, Koblach, Austria). Four hundred and forty-three cuboid-shaped samples with the dimensions of $6.3 \times 6.3 \times 16 \mathrm{~mm}$ were designed and then milled (Ceramill Mind, Ceramill Motion; Amann Girrbach AG, Koblach, Austria). All specimens were sintered for 10 hours (Ceramill Therm; Amann Girrbach AG, Koblach, Austria); the temperature growth was $8^{\circ} \mathrm{C} / \mathrm{min}$ from $200^{\circ} \mathrm{C}$ to $1450^{\circ} \mathrm{C}$, two hours of constant temperature and then the cooling time. The material shrinkage was approximately $21 \%$. Eventually specimens were $5 \times 5 \times 12.6 \mathrm{~mm}$ in dimension.

The specimens were randomly divided into sixteen groups depending on the grade of alumina particles used for air abrasion and on the type of resin cement and primer used (Table 1). One fourth of samples were left untreated. The others were alumina air-abraded with three different grades of alumina particle, $50 \mu \mathrm{m}, 110 \mu \mathrm{m}$ or $250 \mu \mathrm{m}$ (Alustral; Omnident Dental Handelsgesellschaft $\mathrm{mbH}$, Rodgau, Germany). All specimens were air-abraded in the same conditions: at $45^{\circ}$ angle, at $13 \mathrm{~mm}$ distance, at 
the pressure of 3 bar, for 10 seconds (Basic Classic Renfert $\mathrm{GmbH}$, Hilzingen, Germany). The surface of each specimen was cleaned with an oil-free air stream. Alumina air-abrasion was followed by the cementation procedure.

Depending on the group division, for each alumina particle grade, four different resin cements with compatible primers were used. Each primer was applied with a microbrush according to the manufacturer's directions. Respective resin cements were layered using a Teflon mold and light-cured (Flashmax P3; CMS Dental, Copenhagen, Denmark). All specimens were stored in distilled water at temperature $37 \pm 1^{\circ} \mathrm{C}$ for 24 hours.

There were four different resin cements compatible with respective primers containing phosphate monomers used in this study. Their chemical composition disclosed by manufacturers is shown in Tables 2 and 3:
- Primer: Clearfil Ceramic Primer, Cement: Panavia F 2.0 (Kuraray Medical Inc., Sakazu, Kurashiki, Okayama, Japan),

- Primer: Monobond Plus, Cement: Multilink Automix (Ivoclar Vivadent AG, Schaan, Liechtenstein),

- Primer: AZ-Primer, Cement: ResiCem (Shofu Inc., Kyoto, Japan),

- Primer: Z-Prime Plus, Cement: Duo-Link (Bisco Inc., Schaumburg, USA).

\section{SHEAR BOND STRENGTH TESTING}

All specimens were subjected to the shear bond strength test using the Universal Testing Machine (Zwick/ Roell Z005; Zwick GmbH \& Co. KG, Ulm, Germany) at

TABLE 1. Experimental groups

\begin{tabular}{|c|c|c|c|c|}
\hline \multirow[b]{2}{*}{$\begin{array}{l}\text { The grade of alumina used for } \\
\text { air-borne particle abrasion }\end{array}$} & \multicolumn{4}{|c|}{ Primer and resin cement } \\
\hline & $\begin{array}{c}\text { Clearfil Ceramic Primer } \\
+ \\
\text { Panavia F 2.0 }\end{array}$ & $\begin{array}{c}\text { Monobond Plus } \\
+ \\
\text { Multilink Automix }\end{array}$ & $\begin{array}{c}\text { AZ Primer } \\
+ \\
\text { ResiCem }\end{array}$ & $\begin{array}{c}\text { Z-Prime Plus } \\
+ \\
\text { Duo-Link }\end{array}$ \\
\hline- & Panavia 0 & Multilink 0 & ResiCem 0 & Duo-Link 0 \\
\hline $50 \mu \mathrm{m}$ & Panavia 50 & Multilink 50 & ResiCem 50 & Duo-Link 50 \\
\hline $110 \mu \mathrm{m}$ & Panavia 110 & Multilink 110 & ResiCem 110 & Duo-Link 110 \\
\hline $250 \mu \mathrm{m}$ & Panavia 250 & Multilink 250 & ResiCem 250 & Duo-Link 250 \\
\hline
\end{tabular}

TABLE 2. Resin cement chemical composition provided by manufacturers

\begin{tabular}{|l|c|c|}
\hline Resin cement & \multicolumn{1}{|c|}{ Main composition provided by the manufacturer } & \multicolumn{1}{c|}{ Manufacturer } \\
\hline Panavia F 2.0 & $\begin{array}{r}\text { Paste A: 10-Methacryloyloxydecyl dihydrogen phosphate (MDP), hydrophobic aromatic dimethacrylate, } \\
\text { hydrophobic aliphatic dimethacrylate, hydrophilic aliphatic dimethacrylate, silanated silica filler, } \\
\text { silanated colloidal silica, dl-camphorquinone, catalysts, initiators } \\
\begin{array}{r}\text { Paste B: hydrophobic aromatic dimethacrylate, hydrophobic aliphatic dimethacrylate, hydrophilic aliphatic } \\
\text { dimethacrylate, silanated barium glass filler, surface treated sodium fluoride, catalysts, accelerators, pigments }\end{array}\end{array}$ & Kuraray Medical Inc. \\
\hline Multilink Automix & $\begin{array}{r}\text { The monomer matrix is composed of dimethacrylate and hydroxyethylmethacrylate (HEMA). } \\
\text { The inorganic fillers include barium glass, ytterbium trifluoride and spheroid mixed oxide. }\end{array}$ & Ivoclar Vivadent AG \\
\hline ResiCem & $\begin{array}{r}\text { Paste A: UDMA, TEGDMA, fluoroaluminosilicate glass, initiator } \\
\text { Paste B: UDMA, TEGDMA, carboxylic acid monomer, fluoroaluminosilicate glass, initiator }\end{array}$ & Shofu Inc. \\
\hline Duo-Link & $\begin{array}{r}\text { Base: Bis-GMA, TEGDMA, UDMA, glass filler } \\
\text { Catalyst: Bis-GMA, TEGDMA, glass filler }\end{array}$ & Bisco Inc. \\
\hline
\end{tabular}

UDMA - urethane dimethacrylate, TEGDMA - triethylene glycol dimethacrylate, Bis-GMA - bisphenol A-glycidyl methacrylate

TABLE 3. Chemical composition of primers provided by manufacturers

\begin{tabular}{|l|c|c|}
\hline Primer & $\begin{array}{c}\text { Main composition provided by the manufacturer } \\
\text { Clearfil Ceramic Primer }\end{array}$ & $\begin{array}{c}\text { K-methacryloxypropyltrimethoxysilane, 10-methacryloyloxydecyl dihydrogen } \\
\text { phosphate (MDP), ethanol }\end{array}$ \\
\hline Monobond Plus & $\begin{array}{c}\text { Alcohol solution of silane methacrylate, phosphoric acid methacrylate, } \\
\text { sulfide methacrylate }\end{array}$ & Ivoclar Vivadent AG \\
\hline AZ-Primer & Acetone, phosphoric acid monomer & Shofu Inc. \\
\hline Z-Prime Plus & Biphenyl dimethacrylate, hydroxyethyl methacrylate, ethanol & Bisco Inc. \\
\hline
\end{tabular}


$1 \mathrm{~mm} / \mathrm{min}$ crosshead speed to failure. To ensure the even distribution of tension during the test, the crosshead was specially designed to match the specimen.

\section{FRACTOGRAPHIC ANALYSIS}

All specimens subjected to SBS test were assessed at $\times 2.5$ magnification in order to examine the mode of failure (Seliga Auctus; Seliga Microscopes Sp. z o. o., Lodz, Poland). Cohesive, adhesive or mixed failure modes were observed. Random specimens were also evaluated with an electron microscope at magnification $\times 35$ (Scanning Electron Microscope S-4700; Hitachi High-Technologies Corporation, Tokyo, Japan).

\section{STATISTICAL ANALYSIS}

The influence of various modifications of the zirconia surface combining air-borne particle abrasion with alumina particle of different grade and the use of four different resin cements with respective primers on shear bond strength of zirconia-based dental ceramics to resin cements was evaluated with the Wilcoxon signed-rank test and the Kruskal-Wallis one-way analysis of variance, with a level of significance set at 0.05 .

\section{RESULTS}

The mean shear bond strength values (MPa) and standard deviations of all tested groups are shown in Figure 1 and Table 4 . The results of the failure mode assessment are shown in Figure 2, Figure 3 and Table 5. The highest shear bond strength of resin cement to zirconia-based ceramics was obtained for Duo-Link in combination with Z-Prime Plus (8.24-14.15 MPa), the lowest for ResiCem and AZ-Primer (5.26-8.58 MPa). The Wilcoxon signedrank test $(p=0.05)$ revealed significant differences for Z-Prime Plus + Duo-Link and for AZ-Primer + ResiCem when compared to other resin agents. There were no significant differences between Clearfil Ceramic Primer + Panavia F2.0 and Monobond Plus + Multilink Automix groups. The values obtained for these two resin cements were between those obtained for ResiCem and Duo-Link. In groups in which the alumina air-abrasion was omitted, there were no significant differences between the Panavia 0 group and the ResiCem 0 group.

The highest values of the shear bond strength of zirconia-based ceramics to resin cements were obtained for specimens air-abraded with $50 \mu \mathrm{m}$ alumina particles, although the Kruskal-Wallis one-way analysis of variance revealed no significant difference between evaluated grades of alumina particles used for air-abrasion $(p=0.05)$. On the other hand, there was a significant difference in the shear bond strength of 3Y-TZP to resin cements between alumina air-abraded specimens and the specimens in which only primer was used (untreated specimens/control groups). The shear bond strength obtained for specimens without mechanical modification was more than twice as low. The failure mode observed for specimens that were not alumina air-abraded was mainly adhesive, while in sandblasted specimens it was cohesive and mixed.

\section{DISCUSSION}

The results obtained in this study indicate that there were significant differences between groups depending on the resin cement and primer used. The highest SBS values were obtained for Duo-Link and Z-Prime Plus (8.24-14.15 MPa, mean 11.61 MPa), independently of the mechanical modification and compared to other resin cements. The explanation for such results was considered in reference to the chemical composition of resin cements and primers used in the current study. There are two monomers present in the primer Z-Prime Plus which have a chemical affinity for $3 \mathrm{Y}-\mathrm{TZP}$, i.e. $10-\mathrm{MDP}$ and BPDM (biphenyl dimethacrylate). Such a formula may enhance chemical bonding to zirconia-based ceramics and probably is the reason why application of this primer yielded significantly increased SBS values. Both monomers are reactive to $3 \mathrm{Y}-\mathrm{TZP}$, as the MDP compound has two active centers and BPDM has at least two potentially active centers which permit chemical bonding with inert zirconia.

On the other hand, the application of AZ-Primer resulted in decreased SBS values (5.26-8.58 MPa, mean $6.85 \mathrm{MPa})$. This primer is composed of the phosphoric acid monomer and acetone serving as a solvent. MDP alone without any additional agents may be not efficient enough in yielding increased bonding capacity of zirconia to resin cement. 3Y-TZP has a relatively non-polar nature and its structure is polycrystalline without the presence of silica. Therefore, silane coupling compounds are not effective; besides, they are unstable in the acidic environment $[8,13]$. Zirconia-based ceramics are also more stable chemically than the silica containing ceramics, thus they are less prone to water hydrolysis. Also, the Clearfil Ceramic Primer and the Monobond Plus Primer gave lower SBS values. Clearfil Ceramic Primer is composed of solvent, silane coupling compound and MDP agent. It does not incorporate any additional chemical compounds enhancing chemical affinity towards zirconia. Moreover 3-methacryloxypropyltrimethoxysilane may become unstable in the company of acidic MDP. The Monobond Plus Primer acts similarly as the Clearfil Ceramic Primer, as it is composed of silane coupling compounds, phosphoric acid methacrylate and sulfide methacrylate.

Xie et al. and Magne et al. confirm those theoretical considerations; they indicate that experimental primers based on organophosphate and carboxylic acid monomers 
A

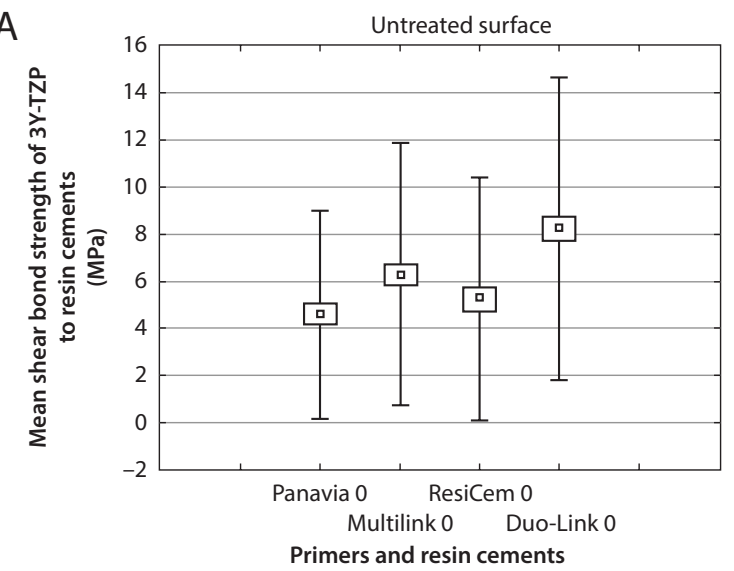

C

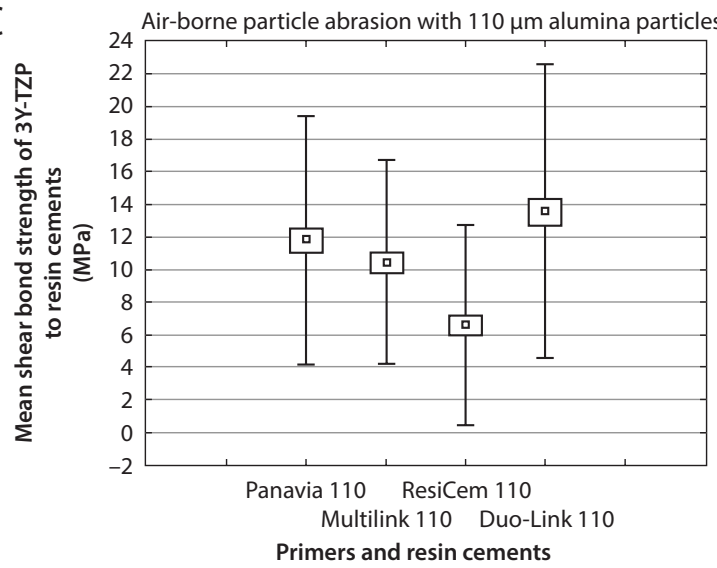

$\mathrm{E}$

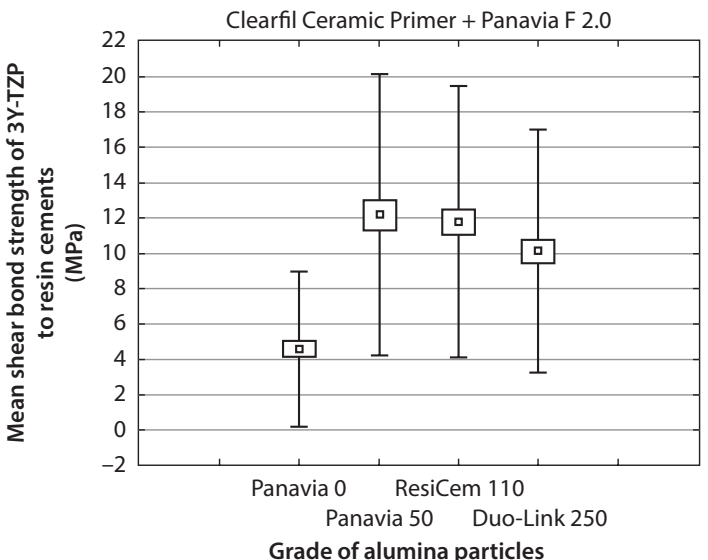

G

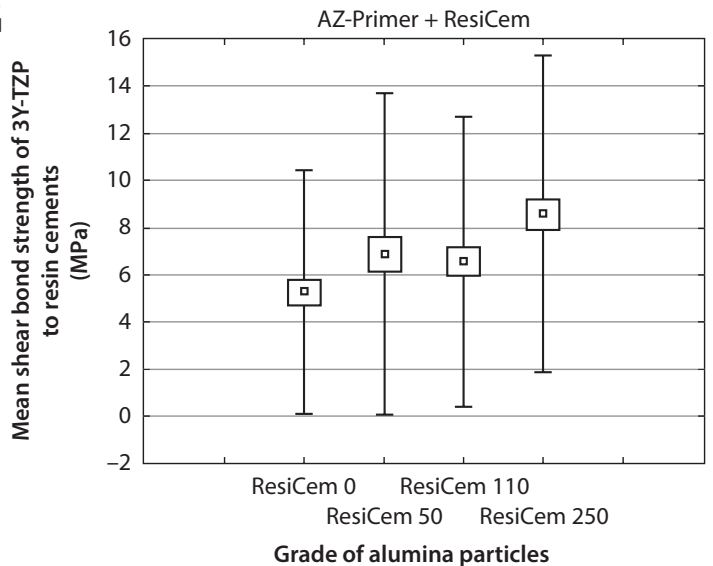

B

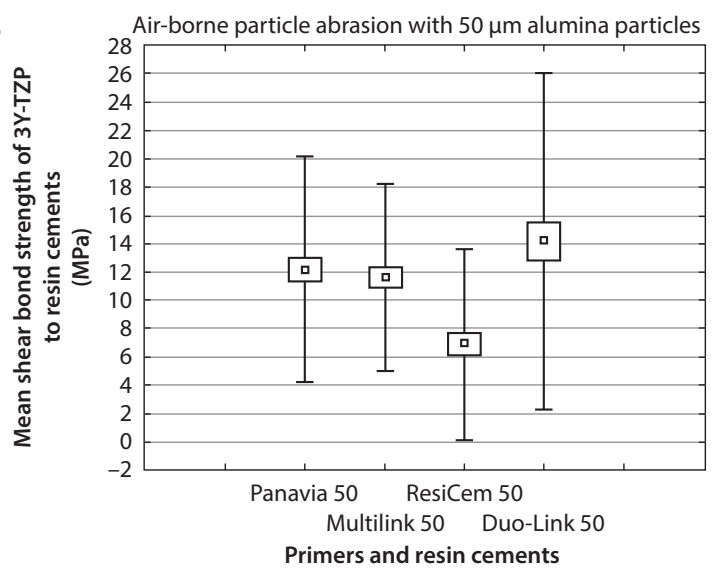

D

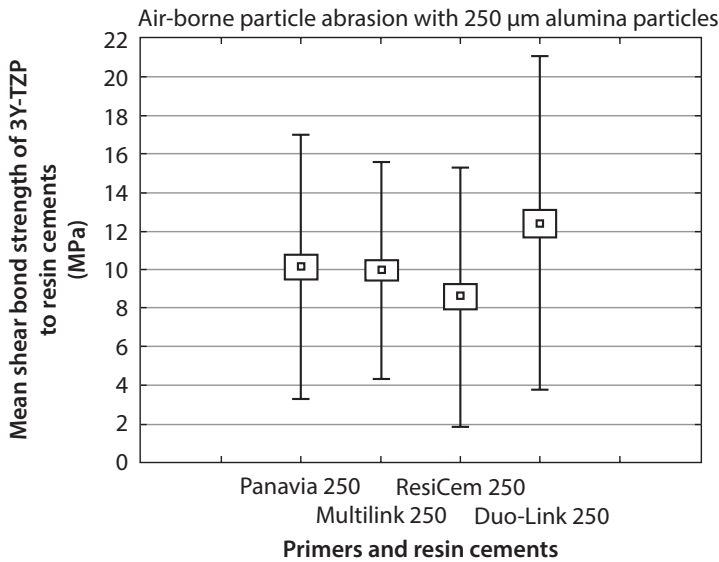

$\mathrm{F}$

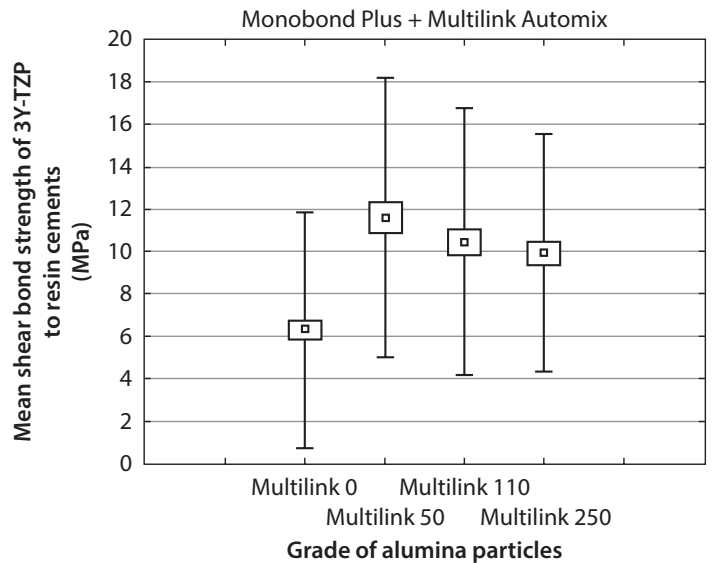

$\mathrm{H}$

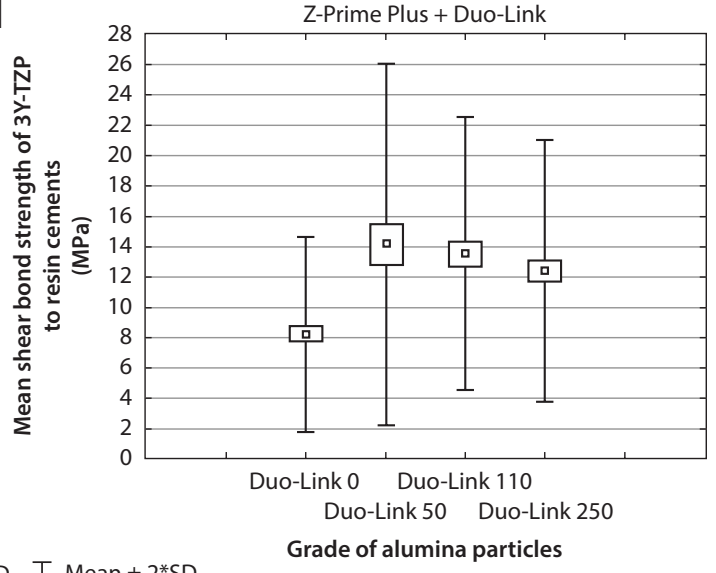

FIGURE 1. Mean shear bond strength values of zirconia to resin cements obtained for 16 experimental groups 
TABLE 4. Mean values of shear bond strength of zirconia-based ceramics to resin cement, standard deviations, medians, maximum and minimum values (MPa) for all tested groups

\begin{tabular}{|c|c|c|c|c|c|}
\hline \multirow{2}{*}{ Groups } & \multicolumn{5}{|c|}{ Shear bond strength (MPa) } \\
\hline & Mean & Standard deviation & Median & Maximum value & Minimum value \\
\hline Panavia 0 & 4.60 & 2.21 & 3.66 & 11.23 & 1.90 \\
\hline Multilink 0 & 6.31 & 2.79 & 5.57 & 14.15 & 3.14 \\
\hline ResiCem 0 & 5.26 & 2.58 & 4.46 & 12.27 & 2.11 \\
\hline Duo-Link 0 & 8.24 & 3.21 & 7.09 & 15.70 & 4.02 \\
\hline Panavia 50 & 12.18 & 3.98 & 12.01 & 21.08 & 5.29 \\
\hline Multilink 50 & 11.59 & 3.30 & 11.59 & 16.84 & 7.78 \\
\hline ResiCem 50 & 6.87 & 3.39 & 5.45 & 13.37 & 3.24 \\
\hline Duo-Link 50 & 14.15 & 5.95 & 12.70 & 29.00 & 7.26 \\
\hline Panavia 110 & 11.79 & 3.83 & 12.32 & 18.25 & 6.31 \\
\hline Multilink 110 & 10.45 & 3.14 & 10.35 & 16.41 & 4.60 \\
\hline ResiCem 110 & 6.57 & 3.07 & 4.95 & 16.98 & 3.90 \\
\hline Duo-Link 110 & 13.55 & 4.50 & 12.90 & 27.59 & 7.78 \\
\hline Panavia 250 & 10.13 & 3.43 & 8.95 & 18.82 & 6.05 \\
\hline Multilink 250 & 9.93 & 2.81 & 9.21 & 15.99 & 5.04 \\
\hline ResiCem 250 & 8.58 & 3.36 & 8.26 & 16.13 & 3.25 \\
\hline Duo-Link 250 & 12.39 & 4.31 & 11.36 & 23.63 & 5.72 \\
\hline
\end{tabular}

TABLE 5. Percentage of failure modes observed after shear bond strength testing

\begin{tabular}{|c|c|c|c|}
\hline \multirow{2}{*}{$\begin{array}{l}\text { Experimental } \\
\text { groups }\end{array}$} & \multicolumn{3}{|c|}{ Failure mode (\%) } \\
\hline & Adhesive & Mixed & Cohesive \\
\hline Panavia 0 & 81 & 19 & 0 \\
\hline Multilink 0 & 39 & 59 & 2 \\
\hline ResiCem 0 & 72 & 28 & 0 \\
\hline Duo-Link 0 & 15 & 80 & 5 \\
\hline Panavia 50 & 10 & 86 & 5 \\
\hline Multilink 50 & 0 & 95 & 5 \\
\hline ResiCem 50 & 10 & 75 & 15 \\
\hline Duo-Link 50 & 0 & 20 & 80 \\
\hline Panavia 110 & 11 & 82 & 7 \\
\hline Multilink 110 & 0 & 88 & 12 \\
\hline ResiCem 110 & 4 & 85 & 12 \\
\hline Duo-Link 110 & 0 & 63 & 37 \\
\hline Panavia 250 & 0 & 89 & 11 \\
\hline Multilink 250 & 4 & 89 & 7 \\
\hline ResiCem 250 & 11 & 85 & 4 \\
\hline Duo-Link 250 & 0 & 43 & 57 \\
\hline
\end{tabular}

increase the resin cement bonding capacity to zirconia-based ceramics. Moreover, they prove that $10-\mathrm{MDP}$ has a chemical affinity for 3Y-TZP and the presence of solvent, such as ethanol, is crucial to create chemical bonds between this compound and zirconia-based ceramics $[13,24]$.

There are only a few research studies that compare the commercial primers and resin cements used in the current study, but their results are similar. Griffin et al. compared the same resin agents and they indicate that the highest SBS values are obtained for Z-Prime Plus and Duo-Link (28.7 MPa), followed by Monobond Plus and Multilink Automix (26.4 MPa), AZ Primer and ResiCem (21.2 MPa), Clearfil Ceramic Primer and Panavia F2.0 (7.5 MPa) [8]. The main difference between the two studies are the results obtained for AZ Primer; however in their study there is a lack of information on the exact air-borne particle abrasion parameters. In the current study, using the air-borne particle abrasion with $250 \mu \mathrm{m}$ alumina particles resulted in higher SBS values when AZ-Primer was applied on the zirconia surface. El-Shrkawy et al. proved that for Multilink Automix resin cement, the additional air-abrasion with $110 \mu \mathrm{m}$ alumina particles resulted in twice as high bond strength values than for untreated zirconia specimens (13.2 MPa vs. 6.1 MPa) [5]. On the other hand, Kitayama et al. compared the bond strength of five different resin cements to zirconia-based ceramics with and without additional primer application; prior to adhesive cementation, they treated the zirconia surface with $70 \mu \mathrm{m}$ alumina air-abrasion. They found that additional primer application resulted in nearly twice as high bond strength values [12]. Samimi et al. assessed the bond strength of zir- 

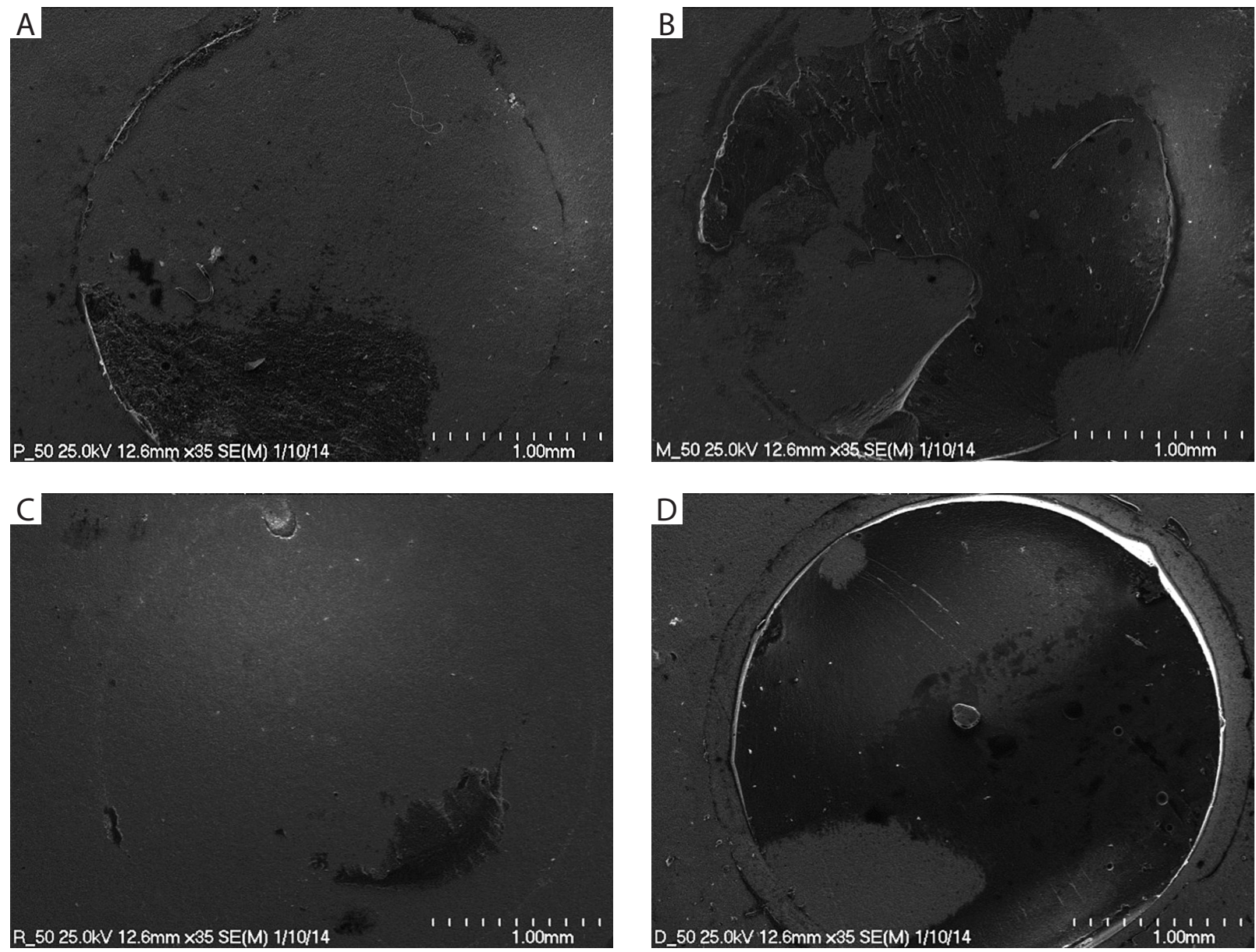

FIGURE 2. Representative SEM micrograph of the failure mode of zirconia surface/resin cement interface at $\times 35$ magnification of specimens treated with air-borne particle abrasion with $50 \mu \mathrm{m}$ alumina particles. A) Panavia F 2.0 and Clearfil Ceramic Primer, B) Multilink Automix and Monobond Plus, C) ResiCem and AZ-Primer, D) Duo-Link and Z-Prime Plus

conia samples to resin cement incorporating the MDP compound (Panavia F 2.0) and to resin cements without organophosphate monomers (Aegis and Esthetic); all samples were alumina air-abraded prior to the cementation procedure. The use of the resin cement incorporating MDP compound resulted in obtaining almost three times higher bond strength values in comparison with resin cements without MDP compound in their composition. Moreover, the use of additional air-borne particle abrasion on the zirconia surface is important as it gave significantly higher bond strength values for Panavia F2.0 than for the Panavia group in which the mechanical modification of the zirconia surface was omitted (28.5 MPa vs. 8.9 $\mathrm{MPa}$ ) [18].

In the current study, omitting the air-borne particle abrasion resulted in much lower SBS values. The same conclusion was drawn by other authors $[2,5,16,18,22$, $25,27]$. Moreover, they confirm not only the lower SBS values of zirconia to resin cements, but also the adhesive interface degradation due to the hydrolysis in case the alumina air-abrasion is omitted $[22,25]$. The obtained results indicate that the optimal adhesive algorithm for resto- rations made of zirconia-based ceramics would be the use of alumina air-borne particle abrasion prior to primer application. Although air-abrasion causes microstructural changes in the topmost layers of zirconia samples such as irregularities, grooves and plastic deformation, as well as tetragonal to monoclinic phase transformation, it has also many advantages $[3,9,10,15,16,19,20,30]$. The positive outcomes of alumina air-abrasion are evident, as it increases bond strength of 3Y-TZP to resin cements, increases flexural strength of $3 \mathrm{Y}-\mathrm{TZP}$, improves marginal adaptation, prevents micro-leakage and removes contaminations $[2,5,7,13,16,18,19,22,25,27,28]$.

The obtained results indicate that omitting airborne alumina abrasion significantly decreases the SBS values of four resin cements to zirconia-based ceramics (mean 6.37 MPa). Treating the zirconia sample surface with air-abrasion resulted in the increase in shear bond strength to resin cements. The highest SBS values were yielded after using $50 \mu \mathrm{m}$ alumina particles (for Panavia F2.0, Multilink Automix, Duo-Link) - $11.59 \mathrm{MPa}$ to $14.15 \mathrm{MPa}$. However, there was no statistical significance manifested in reference to the alumina particle 

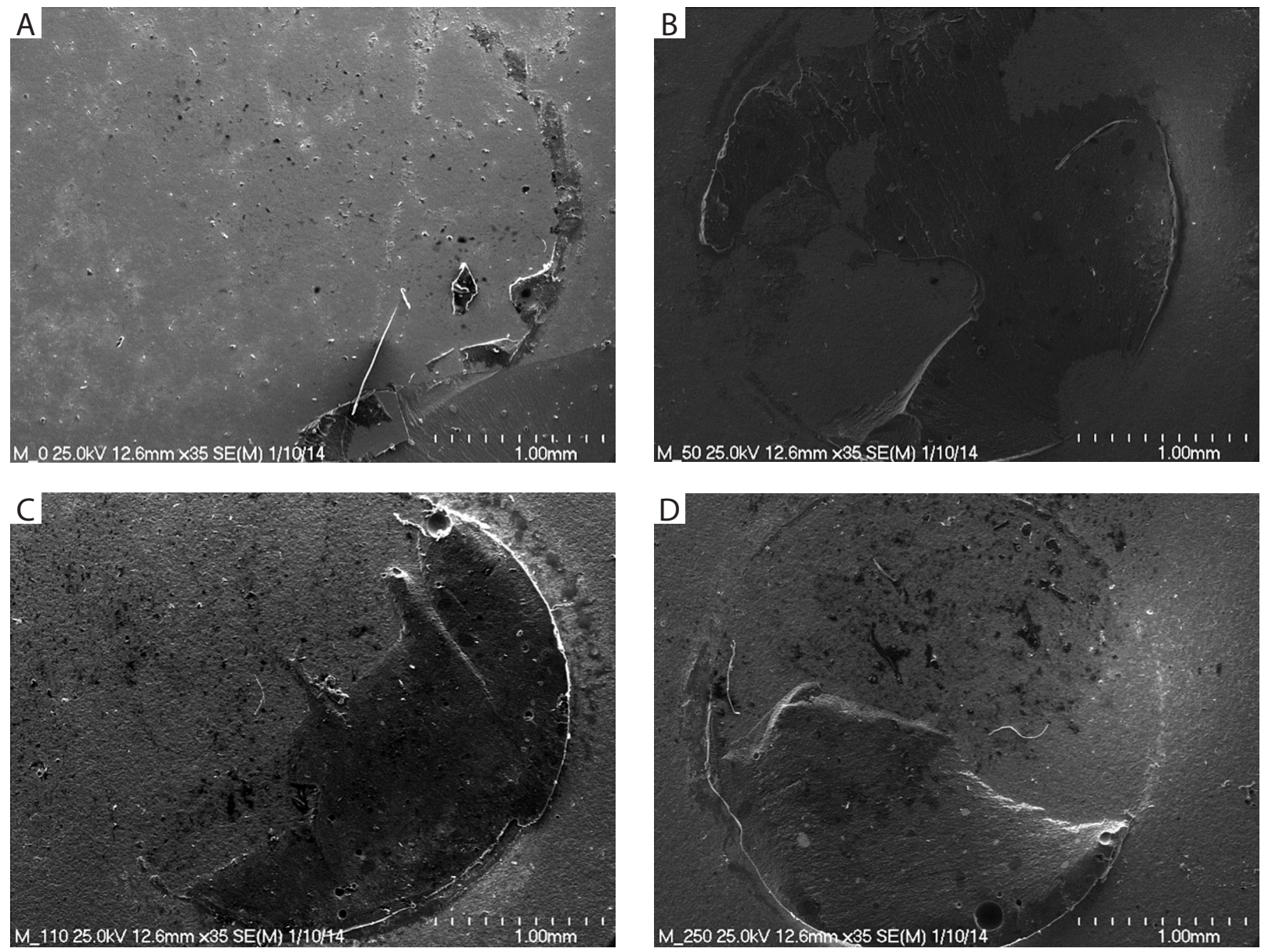

FIGURE 3. Representative SEM micrograph of the failure mode of zirconia surface/resin cement interface at $\times 35$ magnification of specimens treated with air-borne particle abrasion with alumina of different grades: A) untreated, B) $50 \mu \mathrm{m}$ alumina particles, C) $110 \mu \mathrm{m}$ alumina particles and D) $250 \mu \mathrm{m}$ alumina particles, where Monobond Plus and Multilink Automix were used

grade used for air-borne particle abrasion. It is also confirmed by other research studies, although the alumina particle grade may be relevant for long-term durable bonding to zirconia $[7,22]$.

The results obtained in the shear bond strength test correlate with results of fractographic analysis of the fracture mode of the $3 \mathrm{Y}-\mathrm{TZP} /$ resin cement interface. The higher the SBS values were, the more mixed, even cohesive failure modes were observed. Omitting the air-abrasion resulted in low SBS values, and for those specimens the failure mode was mainly adhesive. The failure mode was mixed for specimens that were treated with air-borne alumina abrasion. Moreover, in groups in which Duo-Link and Z-Prime Plus were used, the failure mode was even cohesive, as for those specimens the highest SBS values were obtained. Similar results were obtained by other researchers $[5,12,13]$. It is also worth mentioning that usually mixed and cohesive failure modes yielded after SBS testing occur in nearly $55 \%$ of the research groups. The adhesive failure modes usually are obtained when the SBS values are rather low [4]. The mixed and cohesive failure mode can be considered as clinically favorable, as the adhesive forces exceeded the material cohesion $[1,4,7]$.

Assuming all results obtained in this study, the most favorable cementation procedure that may be applied to zirconia-based ceramic restorations would be a two-stage procedure: first, air-borne particle abrasion with $50 \mu \mathrm{m}$ alumina particles and then application of primer incorporating 10-MDP and carboxylic monomers, such as Z-Prime Plus primer.

\section{CONCLUSIONS}

The chemo-mechanical treatment of zirconia surface before adhesive cementation is crucial to obtain satisfactory values of shear bond strength to resin cements.

The highest shear bond strength of 3Y-TZP to resin cements is obtained after $50 \mu \mathrm{m}$ alumina particle air-abrasion of the zirconia surface, although there is no statistical significance according to the alumina particle grade used for air-abrasion. Omitting alumina air-abrasion results in significantly decreased shear bond strength values. 
The highest shear bond strength of 3Y-TZP to resin cements is obtained for Z-Prime Plus primer and DuoLink resin cement.

\section{CONFLICT OF INTEREST}

The authors declare no potential conflicts of interest with respect to the research, authorship, and/or publication of this article.

\section{References}

1. Bielen V, Inokoshi M, De Munck J, Zhang F, Vanmeensel K, Minakuchi S, Vleugels J, Naert I, Van Meerbeck B. Bonding effectiveness to differently sandblasted dental zirconia. J Adhes Dent 2015; 17: 235-242

2. Blatz MB, Phark J-H, Ozer F, Mante FK, Saleh N, Bergler M, Sadan A. In vitro comparative bond strength of contemporary self-adhesive resin cements to zirconium oxide ceramic with and without air-particle abrasion. Clin Oral Investig 2010; 14 187-192.

3. Chintapalli RK, Marro FG, Jimenez- Pique E, Anglada M. Phase transformation and subsurface damage in 3Y-TZP after sandblasting. Dent Mater 2013; 29: 566-572.

4. Craig's restorative dental materials. Sakaguchi RL, Powers JM. Philadelphia: Elsevier Mosby; 2014, pp. 83-108.

5. El-Shrkawy ZR, El-Hosary MM, Saleh O, Mandour MH. Effect of different surface treatments on bond strength, surface and microscopic structure of zirconia ceramic. Future Dental Journal 2016; 2: 41-53.

6. Fleming GJP, Addison O. Adhesive cementation and the strengthening of all-ceramic dental restorations. Journal of Adhesion Science and Technology 2009; 23: 945-959.

7. Gomes AL, Castillo-Oyague R, Lynch ChD, Montero J, Albaladejo A. Influence of sandblasting granulometry and resin cement composition on microtensile bond strength to zirconia ceramic for dental prosthetic Framework. J Dent 2013; 41: 31-41.

8. Griffin JD, Suh B, Chen L, Brown DJ. Surface treatments for zirconia bonding: A clinical perspective. Canadian Journal of Restorative Dentistry and Prosthodontics 2010; Winter: 23-29.

9. Hallmann L, Ulmer P, Reusser E, Hammerle CHF. Effect of blasting pressure, abrasive particle size and grade on phase transformation and morphological change of dental zirconia surface. Surface and Coatings Technology 2012; 206: 4293-4302.

10. Hallmann L, Ulmer P, Reusser E, Hammerle CHF. Surface characterization of dental Y-TZP ceramic after air abrasion treatment. J Dent 2012; 40: 723-735.

11. Kim MJ, Kim YK, Kim KH, Kwon TY. Shear bond strengths of various luting cements to zirconia ceramic: Surface chemical aspects. J Dent 2011; 39: 795-803.

12. Kitayama S, Nikaido T, Takahashi R, Zhu L, Ikeda M, Foxton RM, Sadr A, Tagami J. Effect of primer treatment on bonding of resin cements to zirconia ceramic. Dent Mater 2010; 26: 426-432.

13. Magne P, Paranhos MPG, Burnett Jr LH. New zirconia primer improves bond strength of resin-based cements. Dent Mater 2010; 26: 345-352.

14. Molin MK, Karlsson SL. Five-year clinical prospective evaluation of zirconia-based Denzir 3-unit FPDs. Int J Prosthodont 2008; 21: 223-227.

15. Monaco C, Tucci A, Esposito L, Scotti R. Microstructural changes produces by abrading $\mathrm{y}$-TZP in presintered and sintered conditions. J Dent 2013; 41: 121-126.

16. Moon J, Kim S, Lee J, Ha S, Choi Y. The effect of preparation order on the crystal structure of yttria-stabilized tetragonal zirconia polycrystal and the shear bond strength of dental resin cements. Dent Mater 2011; 27: 651-663.
17. Sailer I, Fehér A, Filser F, Gauckler LJ, Lüthy H, Hämmerle CH. Five-year clinical results of zirconia frameworks for posterior fixed partial dentures. Int J Prosthodont 2007; 20: 383-388.

18. Samimi P, Hasankhani A, Matinlinna JP, Mirmohammadi H. Effect of adhesive resin type for bonding to zirconia using two surface pretreatments. J Adhes Dent 2015; 17: 353-359.

19. Sato H, Yamadad K, Pezzotti G, Nawa M, Ban S. Mechanical properties of dental zirconia ceramics changed with sandblasting and heat treatment. Dent Mater J 2008; 27: 408-414.

20. Subasi MG, Inan O. Evaluation of the topographical Surface changes and roughness of zirconia after different Surface treatments. Lasers Med Sci 2012; 27: 735-742.

21. Thompson JY, Stoner BR, Piascik JR, Smith R. Adhesion/cementation to zirconia and other non-silicate ceramics: where are we now? Dent Mater 2011; 27: 71-82.

22. Tsuo Y, Yoshida K, Atsuta M. Effects of alumina-blasting and adhesive primers on bonding between resin luting agent and zirconia ceramics. Dent Mater J 2006; 25: 669-674.

23. Uo M, Sjogren G, Sundh A, Goto M, Watari F. Effect of surface condition of dental zirconia ceramic (Denzir) on bonding. Dent Mater J 2006; 25: 626-631.

24. Xie H, Tay FR, Zhang F, Lu Y, Shen S, Chen C. Coupling of 10-methacryloyloxydecyldihydrogen phosphate to tetragonal zirconia: effect of $\mathrm{pH}$ reaction conditions on coordinate bonding. Dent Mater 2015; 31: e218-e225.

25. Yang B, Barloi A, Kern M. Influence of air-abrasion on zirconia ceramic bonding using an adhesive composite resin. Dent Mater 2010; 26: 44-50

26. Yoshida K, Tsuo Y, Atsuta M. Bonding of dual-cured resin cement to zirconia ceramic using phosphate acid estermonomer and zirconate coupler. J Biomed Mater Res B Appl Biomater 2006; 77: 28-33.

27. Yoshida K, Tsuo Y, Meng X, Atsuta M. Mechanical properties of dual-cured resin luting agents for ceramic restoration. J Prosthodont 2007; 16: 370-376.

28. Yun J, Ha S, Lee J, Kim S. Effect of sandblasting and various metal primers on the shear bond strength of resin cement to Y-TZP ceramic. Dent Mater 2010; 26: 650-658.

29. Zarone F, Russo S, Sorrentino R. From porcelain-fused-to-metal to zirconia: clinical and experimental considerations. Dent Mater 2011; 27: 83-96.

30. Zhang Y, Lawn BR, Rekow ED, Thompson VP. Effect of sandblasting on the long term performance of dental ceramics. J Biomed Mater Res B Appl Biomater 2004; 71: 381-386. 